\title{
Combined influence of media use on subjective health in elementary school children in Japan: a population-based study
}

Harunobu Nakamura ${ }^{1 * \dagger}$, Kumiko Ohara ${ }^{1 \dagger}$, Katsuyasu Kouda ${ }^{2 \dagger}$, Yuki Fujita ${ }^{2+}$, Tomoki Mase ${ }^{1,3+}$, Chiemi Miyawaki ${ }^{1 \dagger}$, Yoshimitsu Okita ${ }^{4+}$ and Tetsuya Ishikawa ${ }^{1+}$

\begin{abstract}
Background: In recent years in Japan, electronic games, home computers, and the internet have assumed an important place in people's lives, even for elementary school children. Subjective health complaints have also become a problem among children. In the present study, we investigated the relationship between media use and health status in elementary school children in Japan.

Methods: A cross-sectional school-based population survey was conducted in 2009 with a sample of fourth-, fifth-, and sixth-grade children (age range: 10-12 years old) in elementary schools in Japan $(n=3,464)$. Self-reported health, lifestyle habits, and time spent using media were assessed.

Results: The use of games, television, and personal computers was significantly associated with lifestyle $(p<0.05)$ and subjective health $(p<0.05)$. In addition, the use of games, the use of television, and the use of personal computers were mutually associated. The greater the number of media used for more than 1 hour was, the higher the odds ratio of the association of media use with unhealthy lifestyle and subjective health complaints was. The plural use of these media had stronger associations with unhealthy lifestyle and subjective health complaints.

Conclusions: Game, television, and personal-computer use were mutually associated, and the plural use of these media had stronger associations with unhealthy lifestyle and subjective health complaints. Excessive use of media might be a risk for unhealthy lifestyle and subjective health complaints.
\end{abstract}

Keywords: Children, Media, Lifestyle, Subjective health

\section{Background}

In recent years, innovation in electronic media technologies has progressed dramatically, and electronic games, home computers, and the internet have assumed an important place in the lives of both adults and children, even elementary school children. Rideout et al. reported that the time spent using television (TV), personal computers (PC), and video games has increased from 1999 through 2009 in the United States of America [1]. Olds et al. reported that Australian children aged 10-13 years engage in nearly 4 hours of media use per day [2]. In Japan, the use of electronic media is similar to that in

\footnotetext{
* Correspondence: hal@kobe-u.ac.jp

'Equal contributors

'Graduate School of Human Development and Environment, Kobe University, 3-11 Tsurukabuto, Nada, Kobe, Japan

Full list of author information is available at the end of the article
}

other industrial countries. For example, the mean prevalence rates of televisions and PCs per household were $99.6 \%$ and $76.0 \%$, respectively, in Japan in 2011 [3]. These media provide us with useful information and fulfilment, but the excessive use of them has been identified as a health issue, even for elementary school children. Extensive television viewing tends to be associated with obesity [4-6], sleep problems $[7,8]$, and attention disorders [9]. Prolonged video viewing is related with poor attention span, poor creative imagination, and poor visual memory [10].

Subjective health encompasses the physical, emotional, mental, social and behavioral components of well-being and functioning as perceived by the individual [11-13], and subjective health complaints have become a problem among children. For example, Gobina et al. reported that

\section{Ciomed Central}


Table 1 Descriptive status of media use in elementary school children

\begin{tabular}{|c|c|c|c|c|c|c|c|c|}
\hline & & \multicolumn{3}{|l|}{ Boys } & \multicolumn{3}{|l|}{ Girls } & \multirow{2}{*}{$\begin{array}{l}\text { p value gender / } \\
\text { grades }\end{array}$} \\
\hline & & 4th grade & 5 th grade & 6th grade & 4th grade & 5 th grade & 6th grade & \\
\hline \multirow[t]{5}{*}{ Game } & None & 86 & 91 & 98 & 167 & 195 & 186 & \multirow[t]{5}{*}{$* / \mathrm{ns}$} \\
\hline & Up to $1 \mathrm{hr}$ & 289 & 311 & 315 & 328 & 346 & 337 & \\
\hline & Up to $2 \mathrm{hrs}$ & 85 & 136 & 134 & 42 & 38 & 53 & \\
\hline & 2 hrs or more & 44 & 51 & 65 & 8 & 14 & 18 & \\
\hline & Total & 504 & 589 & 612 & 545 & 593 & 594 & \\
\hline \multirow[t]{5}{*}{ TV } & None & 33 & 33 & 25 & 36 & 26 & 20 & \multirow[t]{5}{*}{$\mathrm{ns} / *$} \\
\hline & Up to $1 \mathrm{hr}$ & 156 & 170 & 164 & 201 & 178 & 148 & \\
\hline & Up to 2 hrs & 147 & 178 & 187 & 151 & 178 & 146 & \\
\hline & 2 hrs or more & 160 & 200 & 226 & 140 & 198 & 275 & \\
\hline & Total & 496 & 581 & 602 & 528 & 580 & 589 & \\
\hline \multirow[t]{5}{*}{ PC } & None & 324 & 331 & 338 & 368 & 332 & 301 & \multirow[t]{5}{*}{$\mathrm{ns} / *$} \\
\hline & Up to $1 \mathrm{hr}$ & 150 & 208 & 216 & 150 & 212 & 228 & \\
\hline & Up to $2 \mathrm{hrs}$ & 14 & 26 & 35 & 15 & 26 & 43 & \\
\hline & 2 hrs or more & 12 & 14 & 15 & 2 & 9 & 14 & \\
\hline & Total & 500 & 579 & 604 & 535 & 579 & 586 & \\
\hline
\end{tabular}

Values are head-counts. TV: television, PC: personal computer.

${ }^{*} p<0.05$ for chi-square analysis.

health complaints, such as headache, stomach-ache, difficulties in getting to sleep, and nervousness were common among adolescents (11-, 13-, and 15-year-old boys and girls) in 19 countries in Europe and in the USA [14]. Ravens-Sieberer et al. reported that $44 \%$ of school children have multiple recurrent health complaints, such as headache, stomach-ache, nervousness, sleeping difficulties, and dizziness, in 41 European and North-American countries. They also reported that older adolescents and girls had more health problems, and the gender difference increased with age [15].

The relation between media use and some aspects of subjective health, such as sleep problems, has been reported $[7,8]$. However, the relation has not been fully elucidated. Since both subjective health complaints and media use are common in adolescents, it is important to determine the relation between media use and the subjective health from a public health perspective. In addition, questions also arise regarding how much media use affects subjective health and what is the effect of the combination of media. However, little evidence has been collected on the influence of media use on children's subjective health.

Therefore, we conducted a population-based crosssectional study to investigate the current status of media use and the association of media use with health status and related factors in elementary school children in Japan.

\section{Methods}

\section{Participants}

We conducted a population-based cross-sectional study in three neighbouring cities (Awaji, Sumoto, and
Minami-awaji) located in the urban area of Hyogo prefecture in the central part of Japan. A selfadministered questionnaire was conducted in May 2009. The subjects were fourth-, fifth-, and sixth-graders (age range: 10-12 years old; mean \pm standard deviation of age: $10.05 \pm 0.81$ years old) in public elementary schools in these three cities $(n=3,690)$. The responders included 3,464 children, and the response rate was $93.9 \%$. The participants included 1,056 fourth-graders ( 509 boys and 547 girls), 1,195 fifth-graders (597 boys and 598 girls), and 1,213 sixth-graders (615 boys and 598 girls). The study was approved by Human Ethics Committee of Graduate School of Human Development and Environment, Kobe University.

\section{Questionnaire}

The questionnaire was anonymous and had three parts and a total of 21 fixed-choice questions. The first part included questions pertaining to the time spent using media (game, TV, and PC). Response options included:" none", " up to $1 \mathrm{hr}$ ", "up to $2 \mathrm{hrs",} \mathrm{"2} \mathrm{hrs} \mathrm{or} \mathrm{more".} \mathrm{The}$ second part included 9 items pertaining to lifestyle, including the following: "bedtime", "waking time", "good feeling on waking", "breakfast eating", "eating breakfast alone", "dinner time", "eating dinner alone", "learning time at home", and "reading at home". The responses for "bedtime" included "-21:00", "21:00-22:00", "22:00-23:00", "23:00-24:00", "24:00-", and "irregular". The response for "waking time" included "-6:00", "6:00-7:00", "7:00-8:00", "8:00-", and "irregular". The responses for "good feeling on walking", "eating breakfast alone", "eating dinner alone", or 
Table 2 Comparative analysis of media use and lifestyle

\begin{tabular}{|c|c|c|c|c|c|c|}
\hline \multirow{2}{*}{ 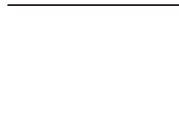 } & \multicolumn{2}{|c|}{ Game } & \multicolumn{2}{|l|}{ TV } & \multicolumn{2}{|l|}{ PC } \\
\hline & $\begin{array}{l}\text { less } \\
\text { than } \\
1 \mathrm{hr}\end{array}$ & $\begin{array}{l}\text { more } \\
\text { than } \\
1 \mathrm{hr}\end{array}$ & $\begin{array}{l}\text { less } \\
\text { than } \\
1 \mathrm{hr}\end{array}$ & $\begin{array}{l}\text { more } \\
\text { than } \\
1 \mathrm{hr}\end{array}$ & $\begin{array}{l}\text { less } \\
\text { than } \\
1 \mathrm{hr}\end{array}$ & $\begin{array}{l}\text { more } \\
\text { than } \\
1 \mathrm{hr}\end{array}$ \\
\hline
\end{tabular}

\begin{tabular}{lllllll}
\hline Bedtime & & & & & & \\
$-21: 00$ & 91 & $13^{*}$ & 57 & $43^{*}$ & 99 & $4^{*}$ \\
21:00-22:00 & 817 & 118 & 409 & 511 & 892 & 30 \\
22:00-23:00 & 964 & 227 & 366 & 808 & 1090 & 84 \\
23:00-24:00 & 210 & 80 & 66 & 217 & 250 & 37 \\
24:00- & 22 & 24 & 10 & 35 & 33 & 11 \\
Irregular & 633 & 226 & 278 & 564 & 782 & 58 \\
Total & 2737 & 688 & 1186 & 2178 & 3146 & 224 \\
Waking time & & & & & & \\
-6:00 & 191 & $30^{*}$ & 97 & $119^{*}$ & 202 & $15^{*}$ \\
6:00-7:00 & 1327 & 287 & 580 & 1003 & 1490 & 98 \\
7:00-8:00 & 944 & 265 & 379 & 814 & 1116 & 74 \\
8:00- & 33 & 15 & 15 & 32 & 38 & 8 \\
Irregular & 234 & 90 & 114 & 202 & 295 & 27 \\
Total & 2729 & 687 & 1185 & 2170 & 3141 & 222
\end{tabular}

Good feeling on waking

$\begin{array}{lllllll}\text { Usually } & 727 & 115^{*} & 356 & 468^{*} & 784 & 41^{*} \\ \text { Often } & 1450 & 316 & 568 & 1170 & 1637 & 108 \\ \text { Occasionally } & 432 & 183 & 207 & 400 & 551 & 52 \\ \text { Seldom } & 89 & 56 & 38 & 102 & 127 & 16 \\ \text { Total } & 2698 & 670 & 1169 & 2140 & 3099 & 217 \\ \text { Breakfast eating } & & & & & & \\ \text { Everyday } & 2455 & 554^{*} & 1069 & 1886^{*} & 2783 & 180^{*} \\ \text { Often } & 194 & 64 & 74 & 179 & 229 & 21 \\ \text { Occasionally } & 58 & 42 & 24 & 74 & 87 & 13 \\ \text { Seldom } & 21 & 23 & 8 & 34 & 33 & 10 \\ \text { Total } & 2728 & 683 & 1175 & 2173 & 3132 & 224\end{array}$

Eating breakfast alone

$\begin{array}{lllllll}\text { Usually } & 353 & 107^{*} & 130 & 321^{*} & 408 & 41^{*} \\ \text { Often } & 440 & 107 & 175 & 361 & 494 & 41 \\ \text { Occasionally } & 471 & 154 & 212 & 408 & 570 & 54 \\ \text { Seldom } & 1457 & 310 & 658 & 1075 & 1655 & 85 \\ \text { Total } & 2721 & 678 & 1175 & 2165 & 3127 & 221 \\ \text { Dinner time } & & & & & & \\ \text {-18:00 } & 101 & 24 & 60 & 63^{*} & 114 & 10 \\ \text { 18:00-19:00 } & 730 & 188 & 342 & 562 & 855 & 46 \\ \text { 19:00-20:00 } & 1383 & 332 & 564 & 1119 & 1577 & 112 \\ \text { 20:00-21:00 } & 440 & 114 & 185 & 358 & 500 & 44 \\ \text { 21:00- } & 54 & 21 & 26 & 50 & 69 & 7 \\ \text { Total } & 2708 & 679 & 1177 & 2152 & 3115 & 219\end{array}$

Eating dinner alone

Usually

68

23
23 $59 \quad 78 \quad 12^{*}$

Table 2 Comparative analysis of media use and lifestyle (Continued)

\begin{tabular}{lllllll}
\hline Often & 235 & 65 & 93 & 202 & 278 & 21 \\
Occasionally & 425 & 127 & 180 & 364 & 494 & 49 \\
Seldom & 1980 & 460 & 867 & 1528 & 2261 & 138 \\
Total & 2708 & 675 & 1171 & 2153 & 3111 & 220 \\
Learning time at home & & & & & \\
none & 78 & $42^{*}$ & 44 & 72 & 103 & $15^{*}$ \\
up to 30 min & 776 & 211 & 358 & 599 & 901 & 63 \\
30-60 min & 1264 & 282 & 528 & 1000 & 1444 & 83 \\
1-2 hrs & 491 & 120 & 198 & 406 & 554 & 50 \\
2 hrs- & 110 & 29 & 50 & 88 & 124 & 11 \\
Total & 2719 & 684 & 1178 & 2165 & 3126 & 222 \\
Reading at home & & & & & & \\
Usually & 341 & $55^{*}$ & 172 & $218^{*}$ & 364 & 30 \\
Often & 1140 & 212 & 494 & 831 & 1245 & 80 \\
Occasionally & 755 & 221 & 328 & 634 & 907 & 55 \\
Seldom & 492 & 195 & 186 & 487 & 618 & 58 \\
Total & 2728 & 683 & 1180 & 2170 & 3134 & 223 \\
\hline
\end{tabular}

Values are head-counts. TV: television, PC: personal computer.

${ }^{*} \mathrm{p}<0.05$ for chi-square test.

"reading at home" included "usually", "often", "occasionally", and "seldom". The responses for "breakfast eating" included "everyday", "often", "occasionally", and "seldom". The responses for "dinner time" included "-18:00", "18:0019:00", "19:00-20:00", "20:00-21:00", and "21:00-". The responses for "learning time at home" included "none", "up to $30 \mathrm{~min} "$, "30-60 min", "1-2 hrs", and "2 hrs-". The third part included 9 items pertaining to subjective health complaints, as follows: "depression", "sleeplessness", "ill at ease", "dizziness", "poor appetite", "headache", "abdominal pain", "short-tempered", and "negative thinking". Each item had 4 responses: "usually", "often", "occasionally", and "seldom". The questionnaire was distributed during class by a teacher at each elementary school, and then collected after it was completed.

\section{Statistical analysis}

To assess the differences among factors, Student's t test and a chi-square test (or Fisher's exact test when appropriate) were performed. Logistic regression analysis was used to evaluate the association between the combined influence of media use and lifestyle habits or subjective health complaints, adjusting for gender and grade. Odds ratios (ORs) and $95 \%$ confidence intervals (95\% CIs) were calculated. Differences with p-values $<0.05$ were considered significant. Statistical analysis was performed by SPSS $^{\circledR} 18.0 \mathrm{~J}$ for Windows (SPSS Inc., Chicago, IL). 
Table 3 Comparative analysis of media use and subjective health complaints

\begin{tabular}{|c|c|c|c|c|c|c|}
\hline & \multicolumn{2}{|c|}{ Game playing } & \multicolumn{2}{|c|}{ TV viewing } & \multicolumn{2}{|c|}{ PC operating } \\
\hline & $\begin{array}{l}\text { less } \\
\text { than } \\
1 \mathrm{hr}\end{array}$ & $\begin{array}{l}\text { more } \\
\text { than } \\
1 \mathrm{hr}\end{array}$ & $\begin{array}{l}\text { less } \\
\text { than } \\
1 \mathrm{hr}\end{array}$ & $\begin{array}{l}\text { more } \\
\text { than } \\
1 \mathrm{hr}\end{array}$ & $\begin{array}{l}\text { less } \\
\text { than } \\
1 \mathrm{hr}\end{array}$ & $\begin{array}{l}\text { more } \\
\text { than } \\
1 \mathrm{hr}\end{array}$ \\
\hline \multicolumn{7}{|l|}{ Depression } \\
\hline Usually & 173 & $82^{*}$ & 77 & 173 & 217 & $33^{*}$ \\
\hline Often & 617 & 189 & 293 & 496 & 739 & 55 \\
\hline Occasionally & 898 & 195 & 361 & 712 & 1006 & 61 \\
\hline Seldom & 1052 & 220 & 458 & 798 & 1186 & 76 \\
\hline Total & 2740 & 686 & 1189 & 2179 & 3148 & 225 \\
\hline \multicolumn{7}{|l|}{ Sleeplessness } \\
\hline Usually & 282 & $118^{*}$ & 131 & $263^{*}$ & 360 & $37^{*}$ \\
\hline Often & 710 & 209 & 320 & 585 & 847 & 57 \\
\hline Occasionally & 745 & 181 & 291 & 615 & 835 & 68 \\
\hline Seldom & 986 & 173 & 435 & 705 & 1087 & 60 \\
\hline Total & 2723 & 681 & 1177 & 2168 & 3129 & 222 \\
\hline \multicolumn{7}{|l|}{ III at ease } \\
\hline Usually & 371 & $153^{*}$ & 173 & $343^{*}$ & 469 & $48^{*}$ \\
\hline Often & 593 & 179 & 238 & 514 & 702 & 53 \\
\hline Occasionally & 788 & 187 & 327 & 631 & 900 & 61 \\
\hline Seldom & 965 & 156 & 439 & 664 & 1051 & 54 \\
\hline Total & 2717 & 675 & 1177 & 2152 & 3122 & 216 \\
\hline \multicolumn{7}{|l|}{ Dizziness } \\
\hline Usually & 125 & $58^{*}$ & 67 & 109 & 160 & $20^{*}$ \\
\hline Often & 385 & 117 & 161 & 333 & 441 & 51 \\
\hline Occasionally & 543 & 130 & 211 & 454 & 625 & 43 \\
\hline Seldom & 1649 & 372 & 728 & 1259 & 1880 & 108 \\
\hline Total & 2702 & 677 & 1167 & 2155 & 3106 & 222 \\
\hline \multicolumn{7}{|l|}{ Poor appetite } \\
\hline Usually & 141 & $50^{*}$ & 59 & $127^{*}$ & 173 & $116^{*}$ \\
\hline Often & 561 & 168 & 231 & 490 & 657 & 62 \\
\hline Occasionally & 840 & 244 & 364 & 704 & 990 & 78 \\
\hline Seldom & 1153 & 216 & 506 & 835 & 1281 & 64 \\
\hline Total & 2695 & 678 & 1160 & 2156 & 3101 & 220 \\
\hline \multicolumn{7}{|l|}{ Headache } \\
\hline Usually & 253 & $87^{*}$ & 129 & 204 & 297 & $36^{*}$ \\
\hline Often & 738 & 206 & 315 & 608 & 856 & 71 \\
\hline Occasionally & 833 & 206 & 342 & 688 & 965 & 61 \\
\hline Seldom & 884 & 179 & 388 & 651 & 993 & 53 \\
\hline Total & 2708 & 678 & 1174 & 2151 & 3111 & 221 \\
\hline \multicolumn{7}{|c|}{ Abdominal pain } \\
\hline Usually & 162 & $58^{*}$ & 73 & 145 & 196 & $24^{*}$ \\
\hline Often & 449 & 139 & 201 & 376 & 532 & 45 \\
\hline Occasionally & 721 & 176 & 302 & 577 & 823 & 60 \\
\hline Seldom & 1354 & 298 & 582 & 1041 & 1538 & 89 \\
\hline
\end{tabular}

Table 3 Comparative analysis of media use and subjective health complaints (Continued)

\begin{tabular}{|c|c|c|c|c|c|c|}
\hline Total & 2686 & 671 & 1158 & 2139 & 3089 & 218 \\
\hline \multicolumn{7}{|c|}{ Short-tempered } \\
\hline Usually & 406 & $151^{*}$ & 172 & $378^{*}$ & 493 & $61^{*}$ \\
\hline Often & 641 & 192 & 246 & 569 & 764 & 53 \\
\hline Occasionally & 848 & 199 & 360 & 670 & 967 & 65 \\
\hline Seldom & 795 & 132 & 377 & 535 & 867 & 42 \\
\hline Total & 2690 & 674 & 1155 & 2152 & 3091 & 221 \\
\hline \multicolumn{7}{|c|}{ Negative thinking } \\
\hline Usually & 257 & $87^{*}$ & 118 & 222 & 297 & $42^{*}$ \\
\hline Often & 712 & 208 & 304 & 592 & 840 & 60 \\
\hline Occasionally & 1005 & 243 & 418 & 806 & 1149 & 76 \\
\hline Seldom & 748 & 145 & 339 & 544 & 842 & 46 \\
\hline Total & 2722 & 683 & 1179 & 2164 & 3128 & 224 \\
\hline
\end{tabular}

Values are head-counts. TV: television, PC: personal computer.

${ }^{*} \mathrm{p}<0.05$ for chi-square test.

\section{Results}

The amounts of time spent using games, TV, and PCs are shown in Table 1 . The time spent using games was significantly less in girls than boys $(\mathrm{p}<0.001)$. In addition, children in older grades spent more time using TV and PCs than children in younger grades (TV, $\mathrm{p}<0.001$; PCs, $\mathrm{p}<0.001)$.

Lifestyle according to time spent using games, TV, or PCs is shown in Table 2. Children who spent more than $1 \mathrm{hr}$ using games had significantly later bedtimes than those who spent less than $1 \mathrm{hr}$ using games $(\mathrm{p}<0.001)$ and had later waking times $(\mathrm{p}<0.001)$, a lower rate of "good feeling on waking" ( $<0.001)$, a lower frequency of eating breakfast $(\mathrm{p}<0.001)$, a higher frequency of eating breakfast alone $(\mathrm{p}<0.001)$, had less learning time at home $(\mathrm{p}<0.001)$, and spent less time reading at home $(\mathrm{p}<0.001)$. Those who spent more than $1 \mathrm{hr}$ using TV had significantly later bedtimes than those who spent less than $1 \mathrm{hr}$ using TV $(\mathrm{p}<0.001)$ and had later waking times $(\mathrm{p}=0.002)$, a lower rate of "good feeling on waking" $(p<0.001)$, a lower frequency of eating breakfast $(\mathrm{p}=0.002)$, a higher frequency of eating breakfast alone $(p=0.001)$, later dinner times $(\mathrm{p}=0.005)$, and spent less time reading at home $(\mathrm{p}<0.001)$. Those who spent more than $1 \mathrm{hr}$ using PCs had significantly later bedtimes than those who spent less

Table 4 Correlation coefficients among game, TV, and PC use

\begin{tabular}{llll}
\hline & Game & TV & PC \\
\hline Game & - & $0.191^{*}$ & $0.242^{*}$ \\
TV & & - & $0.110^{*}$ \\
PC & & - \\
\hline
\end{tabular}

The number is Spearman's $\rho$. TV: television, PC: personal computer. ${ }^{*} p<0.05$ for Spearman's correlation coefficients. 
Table 5 Associations between plural use of media and lifestyles

\begin{tabular}{|c|c|c|c|c|c|c|}
\hline & \multicolumn{3}{|c|}{ not adjusted } & \multicolumn{3}{|c|}{ adjusted* } \\
\hline & Odds & $95 \% \mathrm{Cl}$ & $p$ value & Odds & $95 \% \mathrm{Cl}$ & $\mathrm{p}$ value \\
\hline \multicolumn{7}{|l|}{ Bedtime } \\
\hline all less than $1 \mathrm{hr}$ (ref) & 1 & & & 1 & & \\
\hline one - more than $1 \mathrm{hr}$ & 1.158 & $(0.963-1.393)$ & 0.119 & 1.224 & $(1.015-1.476)$ & 0.034 \\
\hline two- more than $1 \mathrm{hr}$ & 1.871 & $(1.484-2.360)$ & $<0.001$ & 1.908 & $(1.502-2.424)$ & $<0.001$ \\
\hline all more than $1 \mathrm{hr}$ & 2.014 & $(1.302-3.117)$ & 0.002 & 2.258 & $(1.446-3.525)$ & $<0.001$ \\
\hline \multicolumn{7}{|l|}{ Waking time } \\
\hline all less than $1 \mathrm{hr}$ (ref) & 1 & & & 1 & & \\
\hline one - more than $1 \mathrm{hr}$ & 1.038 & $(0.800-1.346)$ & 0.779 & 1.088 & $(0.837-1.415)$ & 0.527 \\
\hline two- more than $1 \mathrm{hr}$ & 1.325 & $(0.954-1.842)$ & 0.093 & 1.286 & $(0.918-1.801)$ & 0.144 \\
\hline all more than $1 \mathrm{hr}$ & 2.328 & $(1.366-3.967)$ & 0.002 & 2.502 & $(1.452-4.312)$ & 0.001 \\
\hline \multicolumn{7}{|l|}{ Good awakened feeling } \\
\hline all less than $1 \mathrm{hr}$ (ref) & 1 & & & 1 & & \\
\hline one - more than $1 \mathrm{hr}$ & 1.123 & $(0.920-1.370)$ & 0.256 & 1.107 & $(0.906-1.352)$ & 0.319 \\
\hline two- more than $1 \mathrm{hr}$ & 2.155 & $(1.690-2.747)$ & $<0.001$ & 2.071 & $(1.617-2.652)$ & $<0.000$ \\
\hline all more than $1 \mathrm{hr}$ & 2.754 & $(1.762-4.304)$ & $<0.001$ & 2.621 & $(1.671-4.111)$ & $<0.000$ \\
\hline \multicolumn{7}{|l|}{ Breakfast eating } \\
\hline all less than $1 \mathrm{hr}$ (ref) & 1 & & & 1 & & \\
\hline one - more than $1 \mathrm{hr}$ & 1.401 & $(0.853-2.301)$ & 0.182 & 1.406 & $(0.855-2.311)$ & 0.179 \\
\hline two- more than $1 \mathrm{hr}$ & 4.168 & $(2.501-6.945)$ & $<0.001$ & 3.843 & $(2.286-6.462)$ & $<0.001$ \\
\hline all more than $1 \mathrm{hr}$ & 8.305 & $(4.222-16.339)$ & $<0.001$ & 7.816 & (3.935-15.525) & $<0.000$ \\
\hline \multicolumn{7}{|l|}{ Eating breakfast alone } \\
\hline all less than $1 \mathrm{hr}$ (ref) & 1 & & & 1 & & \\
\hline one - more than $1 \mathrm{hr}$ & 1.315 & $(1.101-1.570)$ & 0.002 & 1.301 & $(1.089-1.555)$ & 0.004 \\
\hline two- more than $1 \mathrm{hr}$ & 1.489 & $(1.181-1.877)$ & 0.001 & 1.436 & $(1.135-1.818)$ & 0.003 \\
\hline all more than $1 \mathrm{hr}$ & 1.647 & $(1.059-2.563)$ & 0.027 & 1.572 & $(1.007-2.452)$ & 0.046 \\
\hline \multicolumn{7}{|l|}{ Dinner eating } \\
\hline all less than $1 \mathrm{hr}$ (ref) & 1 & & & 1 & & \\
\hline one - more than $1 \mathrm{hr}$ & 0.982 & $(0.800-1.206)$ & 0.865 & 0.979 & $(0.797-1.202)$ & 0.839 \\
\hline two- more than $1 \mathrm{hr}$ & 1.329 & $(1.024-1.725)$ & 0.033 & 1.306 & $(1.001-1.703)$ & 0.049 \\
\hline all more than $1 \mathrm{hr}$ & 1.069 & $(0.624-1.830)$ & 0.808 & 1.048 & $(0.610-1.801)$ & 0.865 \\
\hline \multicolumn{7}{|l|}{ Eating dinner alone } \\
\hline all less than $1 \mathrm{hr}$ (ref) & 1 & & & 1 & & \\
\hline one - more than $1 \mathrm{hr}$ & 1.227 & $(0.952-1.582)$ & 0.115 & 1.220 & $(0.946-1.575)$ & 0.126 \\
\hline two- more than $1 \mathrm{hr}$ & 1.222 & $(0.873-1.711)$ & 0.242 & 1.154 & $(0.820-1.624)$ & 0.412 \\
\hline all more than $1 \mathrm{hr}$ & 1.657 & $(0.920-2.985)$ & 0.092 & 1.563 & $(0.864-2.828)$ & 0.140 \\
\hline \multicolumn{7}{|l|}{ Learning time at home } \\
\hline all less than $1 \mathrm{hr}$ (ref) & 1 & & & 1 & & \\
\hline one - more than $1 \mathrm{hr}$ & 0.923 & $(0.763-1.116)$ & 0.408 & 0.949 & (0.784-1.149) & 0.591 \\
\hline two- more than $1 \mathrm{hr}$ & 0.957 & $(0.740-1.236)$ & 0.735 & 0.908 & $(0.755-1.273)$ & 0.883 \\
\hline all more than $1 \mathrm{hr}$ & 0.629 & $(0.397-0.997)$ & 0.049 & 0.672 & $(0.423-1.069)$ & 0.094 \\
\hline \multicolumn{7}{|l|}{ Reading at home } \\
\hline all less than $1 \mathrm{hr}$ (ref) & 1 & & & 1 & & \\
\hline one - more than $1 \mathrm{hr}$ & 1.356 & $(1.157-1.588)$ & $<0.001$ & 1.360 & $(1.159-1.596)$ & $<0.001$ \\
\hline
\end{tabular}


Table 5 Associations between plural use of media and lifestyles (Continued)

\begin{tabular}{|c|c|c|c|c|c|c|}
\hline two- more than $1 \mathrm{hr}$ & 2.081 & $(1.679-2.580)$ & $<0.001$ & 1.835 & $(1.474-2.285)$ & $<0.001$ \\
\hline all more than $1 \mathrm{hr}$ & 1.807 & $(1.184-2.756)$ & 0.006 & 1.633 & $(1.065-2.504)$ & 0.025 \\
\hline
\end{tabular}

ref: reference.

*adjusted by gender and grade.

than $1 \mathrm{hr}$ using PCs $(\mathrm{p}<0.001)$ and had later waking times $(\mathrm{p}=0.025)$, a lower rate of "good feeling on waking" $(\mathrm{p}=0.005)$, a lower frequency of eating breakfast $(p<0.001)$, a higher frequency of "eating breakfast alone" $(p<0.001)$, a higher frequency of "eating dinner alone" $(\mathrm{p}=0.005)$, and had more "learning time at home" $(\mathrm{p}=0.001)$.

Subjective health complaints according to time spent using games, TV, and PCs are shown in Table 3. Children who spent more than $1 \mathrm{hr}$ using games had a significantly higher frequency of depression than those who spent less than $1 \mathrm{hr}$ using games $(\mathrm{p}<0.001)$ and had a higher frequency of sleeplessness $(\mathrm{p}<0.001)$, feeling ill at ease $(\mathrm{p}<0.001)$, dizziness $(\mathrm{p}<0.001)$, poor appetite $(\mathrm{p}<0.001)$, headache $(\mathrm{p}=0.002)$, abdominal pain $(\mathrm{p}=0.003)$, being short-tempered $(\mathrm{p}<0.001)$, and negative thinking $(\mathrm{p}<0.001)$. Those who spent more than $1 \mathrm{hr}$ using TV had a significantly higher frequency of sleeplessness than those who spent less than $1 \mathrm{hr}$ using TV $(\mathrm{p}=0.032)$ and had a higher frequency of feeling ill at ease $(p=0.001)$, poor appetite $(p=0.037)$, and being short-tempered $(p<0.001)$. Those who spent more than $1 \mathrm{hr}$ using PCs had a significantly higher frequency of depression than those who spent less than $1 \mathrm{hr}$ using PCs $(\mathrm{p}=0.001)$ and had a higher frequency of sleeplessness $(p=0.022)$, feeling ill at ease $(\mathrm{p}=0.008)$, dizziness $(\mathrm{p}<0.001)$, poor appetite $(\mathrm{p}=0.003)$, headache $(\mathrm{p}=0.001)$, abdominal pain $(\mathrm{p}=0.010)$, being short-tempered $(\mathrm{p}<0.001)$, and negative thinking $(\mathrm{p}<0.001)$.

The relationships among games, TV, and PCs are shown in Table 4. Games were positively related with TV (Spearman's $\rho=0.191, \mathrm{p}<0.001$ ) or PCs (Spearman's $\rho=0.242, \mathrm{p}<0.001)$. TV was positively related with PCs (Spearman's $\rho=0.110, \mathrm{p}<0.001$ ).

The results of the logistic regression model exploring the association between the plural use of media and lifestyle are shown in Table 5. Children who spent more than $1 \mathrm{hr}$ on each of one, two, or all media devices were almost twice as likely (odds of one device: 1.224, $\mathrm{p}=0.034$; odds of two devices: $1.908, \mathrm{p}<0.001$; odds of all devices: $2.258, \mathrm{p}<0.001)$ to go to bed at later hours, after the model was adjusted for sex and grade. Those who spent more than $1 \mathrm{hr}$ on each of all media devices were almost two and one-half times more likely (odds: 2.502, $\mathrm{p}=0.001)$ to wake at later hours, after the model was adjusted for sex and grade. Those who spent more than $1 \mathrm{hr}$ on each of two or all media devices were twice as likely (odds of two devices: 2.071, $\mathrm{p}<0.001$ ) or two and one-half times as likely (odds of all devices: 2.621, $\mathrm{p}<0.001$ ) to have a bad feeling on waking, after the model was adjusted for sex and grade. Those who spent more than $1 \mathrm{hr}$ on each of two or all media devices were about 4 times (odds of two devices: $3.843, \mathrm{p}<0.001$ ) or 8 times (odds of all devices: $7.816, \mathrm{p}<0.001)$ more likely to eat breakfast with low frequency, after the model was adjusted for sex and grade. Those who spent more than $1 \mathrm{hr}$ on each of one, two, or all media devices were about one and one-half times more likely (odds of one device: 1.301, $\mathrm{p}=0.004$; odds of two devices: 1.436, $\mathrm{p}=0.003$; odds of all devices: 1.572, $\mathrm{p}=0.046)$ to eat breakfast alone, after the model was adjusted for sex and grade. Those who spent more than $1 \mathrm{hr}$ on each of two or all media devices were about one and one-half times more likely (odds of two devices: 1.436, $\mathrm{p}=0.003$; odds of all devices: $1.572, \mathrm{p}=0.046$ ) to eat breakfast alone, after the model was adjusted for sex and grade. Those who spent more than $1 \mathrm{hr}$ on each of two media devices were almost one and one-half times more likely (odds: 1.306, $\mathrm{p}=0.049$ ) to eat dinner at later hours, after the model was adjusted for sex and grade. Those who spent more than $1 \mathrm{hr}$ on each of one, two, or all media devices were about one and one-half times (odds of one device: 1.360, $\mathrm{p}<0.001$; odds of all devices: $1.633, \mathrm{p}=0.025$ ) or two times (odds of two devices: $1.835, \mathrm{p}<0.001$ ) more likely to read books with low frequency, after the model was adjusted for sex and grade.

The results of the logistic regression model exploring the association between the plural use of media and subjective complaints are shown in Table 6. Children who spent more than $1 \mathrm{hr}$ on each of two or all media devices were almost one and one-half times more likely (odds of two devices: 1.456, $\mathrm{p}=0.001$; odds of all devices: 1.644 , $\mathrm{p}=0.024$ ) to feel "depression", after the model was adjusted for sex and grade. Those who spent more than $1 \mathrm{hr}$ on each of two or all media devices were almost one and one-half times (odds of two devices: 1.477, $\mathrm{p}=0.001$ ) or two times (odds of all devices: 1.783, $\mathrm{p}=0.007$ ) more likely to feel "sleeplessness", after the model was adjusted for sex and grade. Those who spent more than $1 \mathrm{hr}$ on each of one, two, or all media devices were between 1.2 and 2.5 times more likely (odds of one device: 1.286, $\mathrm{p}=0.003$; odds of two devices: $1.704, \mathrm{p}<0.001$; odds of all devices: 2.476, $\mathrm{p}<0.001$ ) to feel "ill at ease", after the model was adjusted for sex and grade. Those who spent more than $1 \mathrm{hr}$ on each of two or all media devices were almost two times more likely (odds of two devices: 1.728, $\mathrm{p}<0.001$; 
Table 6 Associations between plural use of media and subjective health complaints

\begin{tabular}{|c|c|c|c|c|c|c|}
\hline & \multicolumn{3}{|c|}{ not adjusted } & \multicolumn{3}{|c|}{ adjusted $^{*}$} \\
\hline & Odds & $95 \% \mathrm{Cl}$ & $p$ value & Odds & $95 \% \mathrm{Cl}$ & $\mathrm{p}$ value \\
\hline \multicolumn{7}{|l|}{ Depression } \\
\hline all less than $1 \mathrm{hr}$ (ref) & 1 & & & 1 & & \\
\hline one - more than $1 \mathrm{hr}$ & 1.132 & $(0.952-1.345)$ & 0.160 & 1.139 & $(0.958-1.355)$ & 0.139 \\
\hline two- more than $1 \mathrm{hr}$ & 1.472 & $(1.176-1.842)$ & 0.001 & 1.456 & $(1.158-1.830)$ & 0.001 \\
\hline all more than $1 \mathrm{hr}$ & 1.639 & $(1.068-2.518)$ & 0.024 & 1.644 & $(1.067-2.532)$ & 0.024 \\
\hline \multicolumn{7}{|l|}{ Sleeplessness } \\
\hline all less than $1 \mathrm{hr}$ (ref) & 1 & & & 1 & & \\
\hline one - more than $1 \mathrm{hr}$ & 1.037 & $(0.881-1.220)$ & 0.664 & 1.074 & $(0.911-1.265)$ & 0.395 \\
\hline two - more than $1 \mathrm{hr}$ & 1.422 & $(1.147-1.762)$ & 0.001 & 1.477 & $(1.185-1.840)$ & 0.001 \\
\hline all more than $1 \mathrm{hr}$ & 1.631 & $(1.073-2.478)$ & 0.022 & 1.783 & $(1.168-2.721)$ & 0.007 \\
\hline \multicolumn{7}{|l|}{ III at ease } \\
\hline all less than $1 \mathrm{hr}$ (ref) & 1 & & & 1 & & \\
\hline one - more than $1 \mathrm{hr}$ & 1.233 & $(1.045-1.454)$ & 0.013 & 1.286 & $(1.087-1.521)$ & 0.003 \\
\hline two - more than $1 \mathrm{hr}$ & 1.848 & $(1.488-2.297)$ & $<0.001$ & 1.704 & $(1.362-2.131)$ & $<0.001$ \\
\hline all more than $1 \mathrm{hr}$ & 2.452 & $(1.600-3.757)$ & $<0.001$ & 2.476 & $(1.601-3.829)$ & $<0.001$ \\
\hline \multicolumn{7}{|l|}{ Dizziness } \\
\hline all less than $1 \mathrm{hr}$ (ref) & 1 & & & 1 & & \\
\hline one - more than $1 \mathrm{hr}$ & 0.929 & $(0.758-1.138)$ & 0.475 & 0.912 & $(0.744-1.119)$ & 0.378 \\
\hline two - more than $1 \mathrm{hr}$ & 1.622 & $(1.264-2.081)$ & $<0.001$ & 1.728 & $(1.338-2.232)$ & $<0.001$ \\
\hline all more than $1 \mathrm{hr}$ & 1.740 & $(1.091-2.775)$ & 0.020 & 1.791 & $(1.117-2.872)$ & 0.016 \\
\hline \multicolumn{7}{|l|}{ Poor appetite } \\
\hline all less than $1 \mathrm{hr}$ (ref) & 1 & & & 1 & & \\
\hline one - more than $1 \mathrm{hr}$ & 1.235 & $(1.030-1.482)$ & 0.023 & 1.248 & $(1.040-1.498)$ & 0.018 \\
\hline two - more than $1 \mathrm{hr}$ & 1.576 & $(1.246-1.994)$ & $<0.001$ & 1.555 & $(1.224-1.975)$ & $<0.001$ \\
\hline all more than $1 \mathrm{hr}$ & 1.497 & $(0.954-2.349)$ & 0.079 & 1.505 & $(0.956-2.369)$ & 0.077 \\
\hline \multicolumn{7}{|l|}{ Headache } \\
\hline all less than $1 \mathrm{hr}$ (ref) & 1 & & & 1 & & \\
\hline one - more than $1 \mathrm{hr}$ & 0.950 & $(0.807-1.119)$ & 0.539 & 0.973 & $(0.826-1.146)$ & 0.742 \\
\hline two - more than $1 \mathrm{hr}$ & 1.258 & $(1.013-1.561)$ & 0.038 & 1.327 & $(1.064-1.654)$ & 0.012 \\
\hline all more than $1 \mathrm{hr}$ & 1.713 & $(1.125-2.607)$ & 0.012 & 1.854 & $(1.214-2.833)$ & 0.004 \\
\hline \multicolumn{7}{|l|}{ Abdominal pain } \\
\hline all less than $1 \mathrm{hr}$ (ref) & 1 & & & 1 & & \\
\hline one - more than $1 \mathrm{hr}$ & 1.016 & $(0.842-1.226)$ & 0.871 & 1.033 & $(0.855-1.248)$ & 0.735 \\
\hline two - more than $1 \mathrm{hr}$ & 1.394 & $(1.095-1.775)$ & 0.007 & 1.420 & $(1.110-1.817)$ & 0.005 \\
\hline all more than $1 \mathrm{hr}$ & 1.443 & $(0.905-2.301)$ & 0.123 & 1.506 & $(0.941-2.411)$ & 0.088 \\
\hline \multicolumn{7}{|l|}{ Short-tempered } \\
\hline all less than $1 \mathrm{hr}$ (ref) & 1 & & & 1 & & \\
\hline one - more than $1 \mathrm{hr}$ & 1.409 & $(1.195-1.661)$ & $<0.001$ & 1.446 & $(1.225-1.706)$ & $<0.001$ \\
\hline two - more than $1 \mathrm{hr}$ & 2.186 & $(1.759-2.716)$ & $<0.001$ & 2.271 & $(1.819-2.836)$ & $<0.001$ \\
\hline all more than $1 \mathrm{hr}$ & 1.738 & $(1.140-2.651)$ & 0.010 & 1.864 & $(1.218-2.853)$ & 0.004 \\
\hline \multicolumn{7}{|l|}{ Negative thinking } \\
\hline all less than $1 \mathrm{hr}$ (ref) & 1 & & & 1 & & \\
\hline one - more than $1 \mathrm{hr}$ & 1.049 & $(0.890-1.236)$ & 0.566 & 1.069 & $(0.906-1.261)$ & 0.432 \\
\hline
\end{tabular}


Table 6 Associations between plural use of media and subjective health complaints (Continued)

\begin{tabular}{|c|c|c|c|c|c|c|}
\hline two - more than $1 \mathrm{hr}$ & 1.339 & $(1.078-1.662)$ & 0.008 & 1.463 & $(1.172-1.826)$ & 0.001 \\
\hline all more than $1 \mathrm{hr}$ & 1.922 & $(1.264-2.921)$ & 0.002 & 2.128 & $(1.394-3.250)$ & $<0.001$ \\
\hline
\end{tabular}

ref: reference.

*adjusted by gender and grade.

odds of all devices: 1.791, $\mathrm{p}=0.016$ ) to feel "dizziness", after the model was adjusted for sex and grade. Those who spent more than $1 \mathrm{hr}$ on each of one or two media devices were almost one and one-half times more likely (odds of one device:1.248, $\mathrm{p}=0.018$; odds of two devices: $1.555, \mathrm{p}<0.001$ ) to have a "poor appetite", after the model was adjusted for sex and grade. Those who spent more than $1 \mathrm{hr}$ on each of two or all media devices were almost one and one-half times (odds of two devices: 1.327, $\mathrm{p}=0.012$ ) or two times (odds of all devices: 1.854, $\mathrm{p}=0.004$ ) more likely to suffer from "headache", after the model was adjusted for sex and grade. Those who spent more than $1 \mathrm{hr}$ on each of two media devices were almost one and one-half times more likely (odds: 1.420, $\mathrm{p}=0.005$ ) to feel "abdominal pain", after the model was adjusted for sex and grade. Those who spent more than $1 \mathrm{hr}$ on each of one, two, or all media devices were almost one and one-half times (odds of one device:1.446, $\mathrm{p}<0.001$ ) or two times (odds of two devices: 2.271, $\mathrm{p}<0.001$; odds of all devices: 1.864, $\mathrm{p}=0.004$ ) more likely to feel "short-tempered", after the model was adjusted for sex and grade. Those who spent more than $1 \mathrm{hr}$ on each of two or all media devices were almost one and one-half times (odds of two devices: 1.463, $\mathrm{p}=0.001$ ) or two times (odds of all devices: 2.128, $\mathrm{p}<0.001$ ) more likely to experience "negative thinking", after the model was adjusted for sex and grade.

\section{Discussion}

We investigated the relationship between media use and lifestyle or subjective health complaints in elementary school children in Japan. The main finding of the present study was that those who spent more time using media had less healthy lifestyles and more subjective health complaints. In addition, game use, TV use, and PC use were mutually associated, and the plural use of these media had a stronger association with unhealthy lifestyles and subjective health complaints.

In the present study, those who spent more than $1 \mathrm{hr}$ using a media device went to bed at a later time, woke at a later time, had a low frequency of feeling good on waking, ate breakfast with low frequency, and ate breakfast alone with high frequency. These indicators related to sleep or breakfast are associated with lifestyle regularity. According to the previous study, there was some evidence indicating that media use consumed time or displaced other activities after school. Van den Bulck reported that secondary school students who watched more television, played computer games, or used the internet went to bed later [16], and skipped meals $[17,18]$. These results suggest that media use may be responsible for lifestyle irregularity through the displacement of daily activities or through surplus time spent.

Regarding subjective health complaints, those who spent more than $1 \mathrm{hr}$ using a media device were more likely to have subjective health complaints, regardless of the kind of media. Bener et al. and Mathers et al. showed similar results in 6- to 18-year-old children and adolescents (mean age, 16 years old) [19,20]. Namely, they found that spending prolonged hours using a computer or TV was associated with poor life habits, such as sleeplessness $[19,20]$. Nagane et al. reported that lifestyle irregularity is associated with health complaints in university students [21]. In the present study, excessive media use was related with unhealthy lifestyles, which may be causally related to the frequency of subjective health complaints. In addition, the present results showed that game and PC use had stronger associations with multiple subjective health complaints than TV viewing, although the prevalence of the excessive use of TV was greater than that for games or PCs. The reason for this discrepancy among these media is not clear. There have been few previous reports in which differences among media are discussed. The present study also cannot address the reason for this discrepancy. It has been reported in children and adolescents that TV viewing is related with sedentary behavior and obesity because it involves long periods of sitting [19,22,23]. On the other hand, most of the studies regarding addiction referred to the influence of game or computer use [24-27]. It is not clear why specific health issues are connected to specific media. The results of the present and previous studies may reflect the mechanism underlying the related health issues. Indeed, games, TV, and PCs involve very different communication methods. For example, TV watching is a one-way communication experience, while game-playing and PC operation involve two-way communication. In future studies, we should focus on the differences among these media.

Game use, TV use, and PC use for more than $1 \mathrm{hr}$ per day were positively mutually correlated. We then verified the cumulative effects when these media were used for more than $1 \mathrm{hr}$ combined. It was found that the greater the number of media used for more than $1 \mathrm{hr}$ was, the higher the odds ratio of the association between media use and subjective health complaints was. Up to now, there has been little evidence with 
which to assess the combined effect of media on subjective health complaints in elementary school children. In adolescents, Punamaki et al. found that intensive usage of information and communication technology was associated with poor subjective health in adolescents [8]. In the present study, the plural use of media was also strongly associated with unhealthy lifestyles. This unhealthy lifestyle that accompanies the plural use of media may have an influence on subjective health complaints. As a consequence, the present results suggest that the plural use of media has a cumulative influence on subjective health.

The limitations of this study should be noted. First, the present study has a cross-sectional design which cannot draw conclusion about any cause-effect relationship. Second, the samples were collected from a limited area in Japan. Future studies will need to collect samples from a wider area and to employ a longitudinal design for the estimation of any cause-effect relationship. Third, the questionnaire in the present study has not been sufficiently validated, and the link between subjective health and objectively assessed health indices has not been established. In addition, we did not separate sedentary gaming from active gaming. Active gaming could be health enhancing, such as through energy expenditure [28]. Therefore, interpretation of our results may be limited.

\section{Conclusions}

We investigated the relationship between media use and lifestyle or subjective health complaints in elementary school children in Japan. Media use was positively associated with unhealthy lifestyles and subjective health complaints. In addition, game, TV, and PC use were mutually associated, and the plural use of these media had stronger associations with unhealthy lifestyles and subjective health complaints.

\section{Competing interests}

The authors declare that they have no competing interests.

\section{Acknowledgements}

We thank the children who participated in the present study. We also thank the many schools and education boards that allowed us to survey the children. We acknowledge the work of all of the teachers who conducted the data collection.

\section{Author details}

'Graduate School of Human Development and Environment, Kobe University, 3-11 Tsurukabuto, Nada, Kobe, Japan. ${ }^{2}$ Department of Public Health, Kinki University Faculty of Medicine, 377-2 Ohno-higashi, Osaka-sayama, Japan. ${ }^{3}$ Department of Childhood Education, Nagoya Women's University, 1302 Takamiya, Tenpaku, Nagoya, Japan. ${ }^{4}$ Graduate School of Science and Technology, Shizuoka University, 3-5-1 Johoku, Naka, Hamamatsu, Japan.

\section{Authors' contributions}

All authors were involved with the study design. $\mathrm{HN}, \mathrm{KO}$, and Tl collected the data. HN, KK, and YF analyzed the data. HN drafted the manuscript with contributions from YO, TM, and CM. All authors read and approved the manuscript.
Received: 11 January 2012 Accepted: 13 June 2012

Published: 13 June 2012

\section{References}

1. Rideout V, Foehr U, Roberts D: Media in the lives of 8-18 year-olds. In Generation M. Menlo Park, CA: Henry J. Kaiser Family Foundation; 2010.

2. Olds T, Ridley K, Dollman J: Screenieboppers and extreme screenies: the place of screen time in the time budgets of 10-13 year-old Australian children. Aust N Z J Public Health 2006, 30(2):137-142.

3. Cabinet Office Government of Japan: Explanation of Monthly Consumer Confidence Survey Covering All of Japan.Tokyo: National Printing Bureau; 2011.

4. Perez A, Hoelscher DM, Springer AE, Brown HS, Barroso CS, Kelder SH, Castrucci BC: Physical activity, watching television, and the risk of obesity in students, Texas, 2004-2005. Prev Chronic Dis 2011, 8(3):A61.

5. Anderson SE, Whitaker RC: Household routines and obesity in US preschool-aged children. Pediatrics 2010, 125(3):420-428.

6. Vandewater EA, Shim MS, Caplovitz AG: Linking obesity and activity level with children's television and video game use. J Adolesc 2004, 27(1):71-85.

7. Gaina A, Sekine M, Hamanishi S, Chen X, Wang H, Yamagami T, Kagamimori S: Daytime Sleepiness and Associated Factors in Japanese School Children. J Pediatr 2007, 151(5):518-522. e514.

8. Punamaki RL, Wallenius M, Nygard CH, Saarni L, Rimpela A: Use of information and communication technology (ICT) and perceived health in adolescence: the role of sleeping habits and waking-time tiredness. $J$ Adolesc 2007, 30(4):569-585.

9. Swing EL, Gentile DA, Anderson CA, Walsh DA: Television and video game exposure and the development of attention problems. Pediatrics 2010, 126(2):214-221.

10. Kumari S, Ahuja S: Video Viewing and Cognitive Development in Preadolescents. Soc Sci Comput Rev 2010, 28(2):170-176.

11. Bullinger M: Assessing health related quality of life in medicine. An overview over concepts, methods and applications in international research. Restor Neurol Neurosci 2002, 20(3-4):93-101.

12. Taillefer M-C, Dupuis G, Roberge M-A, LeMay S: Health-Related Quality of Life Models: Systematic Review of the Literature. Sol Indic Res 2003, 64(2):293-323

13. The World Health Organization Quality of Life assessment (WHOQOL): position paper from the World Health Organization. Soc Sci Med 1995, 41(10):1403-1409.

14. Gobina I, Valimaa R, Tynjala J, Villberg J, Villerusa A, lannotti RJ, Godeau E, Gabhainn SN, Andersen A, Holstein BE, et al: The medicine use and corresponding subjective health complaints among adolescents, a cross-national survey. Pharmacoepidemiol Drug Saf 2011, 20(4):424-431.

15. Ravens-Sieberer U, Torsheim T, Hetland J, Vollebergh W, Cavallo F, Jericek H, Alikasifoglu M, Valimaa R, Ottova V, Erhart M: Subjective health, symptom load and quality of life of children and adolescents in Europe. Int J Public Health 2009, 54(Suppl 2):151-159.

16. Van den Bulck J: Television viewing, computer game playing, and Internet use and self-reported time to bed and time out of bed in secondary-school children. Sleep 2004, 27(1):101-104.

17. Van den Bulck J, Eggermont S: Media use as a reason for meal skipping and fast eating in secondary school children. J Hum Nutr Diet 2006, 19(2):91-100.

18. Custers K, Van den Bulck J: Television viewing, computer game play and book reading during meals are predictors of meal skipping in a cross-sectional sample of 12-, 14- and 16-year-olds. Public Health Nutr 2010, 13(4):537-543.

19. Bener A, Al-Mahdi HS, Vachhani PJ, Al-Nufal M, Ali Al: Do excessive internet use, television viewing and poor lifestyle habits affect low vision in school children?. Journal of child health care: for professionals working with children in the hospital and community 2010, 14(4):375-385.

20. Mathers M, Canterford L, Olds T, Hesketh K, Ridley K, Wake M: Electronic media use and adolescent health and well-being: cross-sectional community study. Acad Pediatr 2009, 9(5):307-314.

21. Nagane M, Suge R, Watanabe SI: Relationship between psychosomatic complaints and circadian rhythm irregularity assessed by salivary levels of melatonin and growth hormone. Journal of circadian rhythms 2011, 9(1):9.

22. Bener A, Al-Mahdi HS, Ali Al, Al-Nufal M, Vachhani PJ, Tewfik I: Obesity and low vision as a result of excessive Internet use and television viewing. Int J Food Sci Nutr 2011, 62(1):60-62. 
23. Fulton JE, Wang X, Yore MM, Carlson SA, Galuska DA, Caspersen CJ: Television viewing, computer use, and BMI among U.S. children and adolescents. J Phys Act Health 2009, 6(Suppl 1):S28-35.

24. Gentile DA, Choo H, Liau A, Sim T, Li D, Fung D, Khoo A: Pathological video game use among youths: a two-year longitudinal study. Pediatrics 2011, 127(2):e319-329.

25. Chiu SI, Lee JZ, Huang DH: Video game addiction in children and teenagers in Taiwan. Cyberpsychol Behav 2004, 7(5):571-581.

26. Skoric MM, Teo LL, Neo RL: Children and video games: addiction, engagement, and scholastic achievement. Cyberpsychol Behav 2009, 12(5):567-572.

27. Gentile D: Pathological video-game use among youth ages 8 to 18 : a national study. Psychological science 2009, 20(5):594-602.

28. White K, Schofield G, Kilding AE: Energy expended by boys playing active video games. J Sci Med Sport 2011, 14(2):130-134.

doi:10.1186/1471-2458-12-432

Cite this article as: Nakamura et al:: Combined influence of media use on subjective health in elementary school children in Japan: a population-based study. BMC Public Health 2012 12:432.

\section{Submit your next manuscript to BioMed Central and take full advantage of:}

- Convenient online submission

- Thorough peer review

- No space constraints or color figure charges

- Immediate publication on acceptance

- Inclusion in PubMed, CAS, Scopus and Google Scholar

- Research which is freely available for redistribution 